\title{
The Quest to Derive Keratinocytes from Pluripotent Stem Cells
}

\author{
James G. Rheinwald
}

Published online: 31 March 2013

(c) Springer Science+Business Media New York 2013

\begin{abstract}
The derivation of keratinocytes from pluripotent stem cells (PSCs) is a worthy goal. Epidermal and corneal keratinocytes cultured from normal human tissue have been very well-characterized for their growth and differentiation properties and their ability to regenerate permanent tissue as autologous transplants. PSC-derived keratinocytes would therefore be ideal for establishing proof-of-principle that normal, well-defined, and functional somatic cell types can be derived from PSCs and used successfully in tissue replacement therapies. Much progress has been made to identify experimental protocols for deriving cells that display some keratinocyte properties from ES and iPS cells. However, the proliferative potential of these cells typically is very limited and many features of these cells are common to all members of the large family of p63 epithelial cell types. Thus many lines of investigation remain to be examined more thoroughly in this important and exciting field.
\end{abstract}

Keywords Epithelial $\cdot$ p63 $\cdot$ Embryonic $\cdot$ Ectoderm · Cultured cells $\cdot$ Keratin

\section{Introduction}

The cultivation of pluripotent embryonic stem (ES) cell lines from mice $[1,2]$ became the basis for gene knockout technology, which supports much of current experimental

\footnotetext{
J. G. Rheinwald ( $\square)$

Department of Dermatology and Harvard Skin Disease Research Center, Brigham and Women's Hospital and Harvard Medical School, HIM 664, 77 Ave. Louis Pasteur, Boston, MA 02115, USA

e-mail: jimrheinwald@gmail.com
}

biology. Generation of similar cell lines from human embryos [3, 4] engendered interest in the idea of inducing these cells to differentiate in culture into clinically useful adult somatic cell types, especially those that are postmitotic in the adult and may deteriorate or become damaged, resulting in severe pathologies. With the discovery that transient expression of four factors can reprogram somatic cells such as skin fibroblasts to a pluripotent state similar to that of ES cells [5-8] ("induced pluripotent stem (iPS) cells" came the prospect of producing autologous somatic cell types from iPS cells for therapeutic transplantation [9]. The subject of this review is research aimed at producing epidermal and corneal keratinocytes from ES and iPS cells. There is no pressing clinical need for PSC-derived keratinocytes, since autologous tissue-derived keratinocytes can be expanded rapidly in culture and transplanted to provide permanent epidermal coverage of full-thickness burn wounds $[10,11]$ and corneal restoration in cases of chemical damage to the eye surface [12]. However, more is known about the keratinocyte than any other cultivable cell type, with respect to growth regulation, protein expression, differentiation, and methods of transplantation to regenerate permanent tissue. It therefore is the ideal cell type for proofof-principle that authentic, normal, and functional adult somatic cell types can be generated from pluripotent stem cells (PSCs).

More than a dozen research groups have reported generating keratinocytes from PSCs during the past several years, so this is an appropriate time for a comprehensive review. The author of this review devoted four years to this effort and appreciates the challenges of finding effective and reproducible protocols to generate keratinocytes from PSC and characterizing them appropriately. With the objective of encouraging research to advance the field, this is a critical review. As is true for all PSC research, scientists 
from many disciplines have been attracted to this particular endeavor. A consequence of this is that progress has been impeded by insufficient awareness of concepts from classical developmental biology and from contemporary comparative epithelial cell biology. A major problem is an unwarranted interpretation of marker expression to identify epithelial cells derived from PSC cells as "keratinocytes". The modest proliferative potential and undetermined growth regulatory characteristics of PSC-derived epithelial cells raises questions about how normal they are and, therefore, their potential value for tissue replacement. The propensity of many cell types of the large family of epithelia that express p63 to undergo epidermoid histogenesis in the organotypic culture assay for tissue-forming potential also has led to premature conclusions about cell type identity. These issues will be discussed at the end of the article, with suggestions for future research.

Properties of Somatic Tissue-Derived Keratinocytes in Culture

A compelling rationale for generating keratinocytes from PSC cells is that so much is known about the behavior of human tissue-derived keratinocytes. Thus the properties of PSC-derived cells can be compared with those of primary keratinocyte cell lines. A culture system employing co-culture with lethally irradiated 3T3 fibroblast feeder cells and use of a mixed DMEM+F12 nutrient medium supplemented with serum, hydrocortisone, EGF, cholera toxin, insulin, thyroxine, and adenine was developed to promote clonal growth and long-term [25-80 population doublings (PDs)] growth of normal human epidermal keratinocytes [13-15]. This culture system supports equally well the growth of keratinocyte subtypes that form other stratified squamous epithelia (i.e., oral, esophageal, corneal, conjunctival, vaginal, and exocervical epithelium) as well as a diverse set of other epithelial cell types, including mammary, tracheobronchial, and bladder urothelial cells [15, 16•, 17-20]. Semi-defined and defined media were developed subsequently that promote excellent clonal growth and serial propagation of keratinocytes without feeder cells, achieved by optimizing amino acid and vitamin concentrations and substituting bovine pituitary extract (BPE) for serum [21-23]. One of these that is widely used is K-sfm (GIBCO/ Invitrogen/Life Technologies), a published modification [22] of an earlier Ham's MCDB150 series medium [21, 23].

Normal human keratinocytes grow well in $\left[\mathrm{Ca}^{2+}\right]$ as high as $1.0 \mathrm{mM}$, producing some stratifying, terminally differentiated cells while retaining a basal layer of proliferative cells [13, 21, 24, 25]. Primary murine epidermal keratinocytes require culture in media containing $<0.1 \mathrm{mM}$ $\mathrm{Ca}^{2+}$ to maintain basal layer-like, proliferation potential, since higher $\left[\mathrm{Ca}^{2+}\right]$ triggers permanent growth arrest and expression of terminal differentiation markers in most cells [26]. Murine keratinocytes can be serially propagated in low $\left[\mathrm{Ca}^{2+}\right]$ versions of the FAD $+3 \mathrm{~T} 3$ feeder system [27] or of K-sfm medium [28•]. The FAD + fibroblast feeder system and K-sfm medium have been used in most protocols aimed at generating keratinocytes from PSCs.

Major markers of basal keratinocytes, which comprise the stem cell compartment of stratified squamous epithelia, are $\Delta \mathrm{Np} 63 \alpha, \mathrm{K} 5 / \mathrm{K} 14$ [29], $\alpha 6 \beta 4$ integrin, and the Lam $\alpha 3$, Lam $\beta 3$, and Lam $\gamma 2$ subunits of the basal lamina protein Laminin-332. The many stratified squamous epithelia differ from one another with respect to additional keratins made by the basal cells and by the set of proteins involved in structural integrity expressed by the terminally differentiated, suprabasal stratified cells. These include the specialized keratin pairs $\mathrm{K} 1 / \mathrm{K} 10$ by the epidermis and also expressed by exocervix and gingiva, K4/K13 by so-called non-keratinizing stratified squamous epithelia such as esophagus, oral buccal and floor of mouth mucosa, and conjunctiva, and K3/K12 by the cornea [30]. Additional suprabasal differentiation proteins are the cornified envelope proteins Involucrin (IVN) [24] and Loricrin (LOR) [31] and the K1/K10 filament aggregating protein Filaggrin (FIL) [32].

Serial culture and xenograft transplantation studies have demonstrated clearly that human keratinocyte subtypes are intrinsically distinct from one another and maintain their identity and preferred pattern of histogenesis in the absence of any specific signals from regional fibroblasts [18, 19, 33]. An "organotypic" culture system, developed to promote formation of an optimally differentiated epidermis from cultured human epidermal keratinocytes [34], instigates some K1/K10 expression and epidermal-like stratum corneum (sc) formation in all keratinocyte subtypes [33] as well as in other members of the p63+ family of epithelial cell types [16•]. This generally unrecognized limitation of standard organotypic culture for determining epithelial cell identity is important in evaluating reports claiming epidermal keratinocyte identity of PSC-derived epithelial cells. Finally, gene expression profiling to compare PSCderived epithelial cells with normal somatic keratinocytes and other epithelial cell types to determine cell type identity and normality generally has not been used.

\section{Overview of the Literature}

The literature on deriving cells exhibiting features of the keratinocyte lineage is summarized in Tables 1 and 2. The highlights are described in the following text.

\section{Murine ES-Derived Epithelial Cells}

The first report of possible conversion of murine ES (mES) cells to keratinocytes was by Bagutti, Watt, and colleagues 


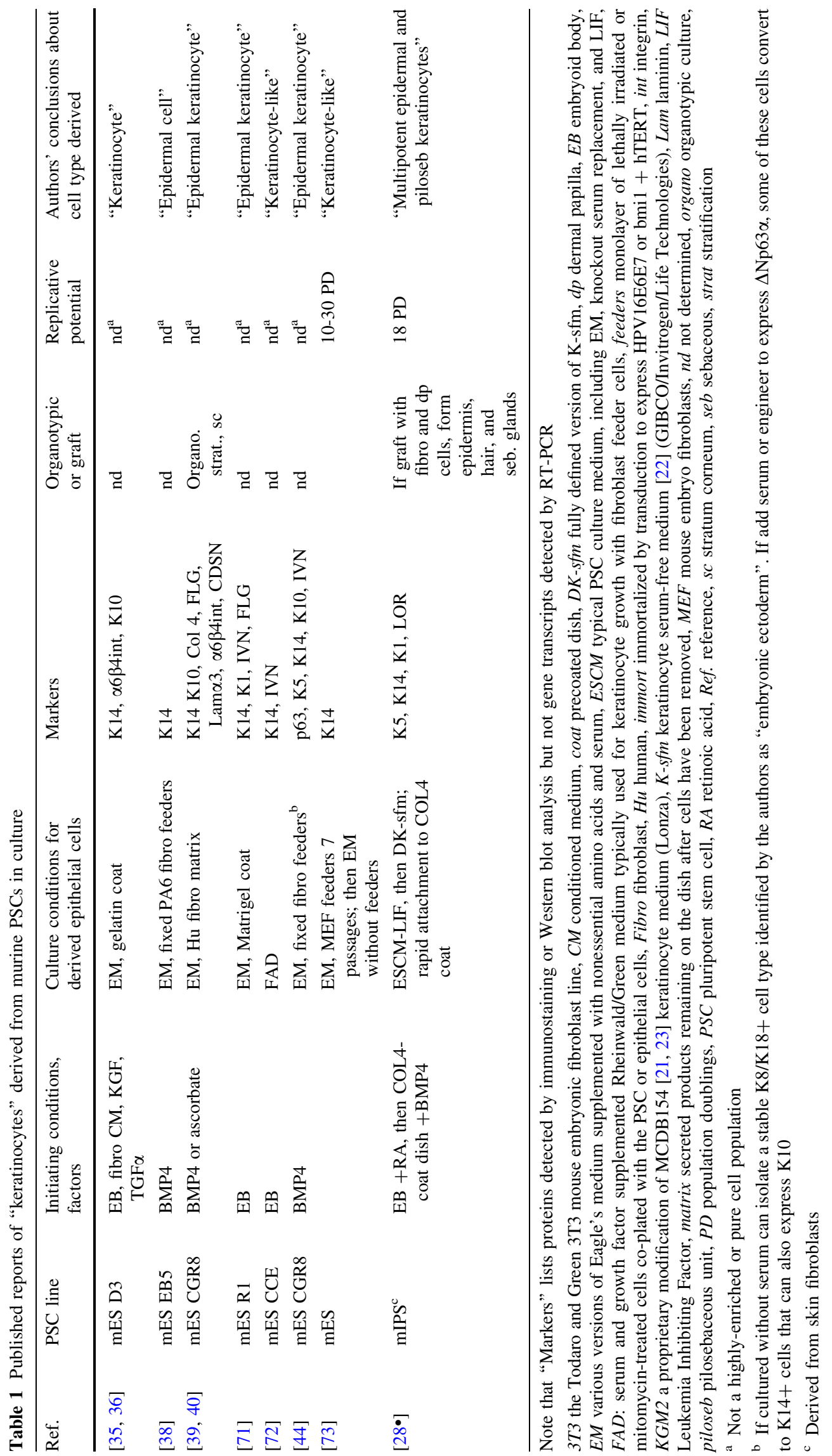




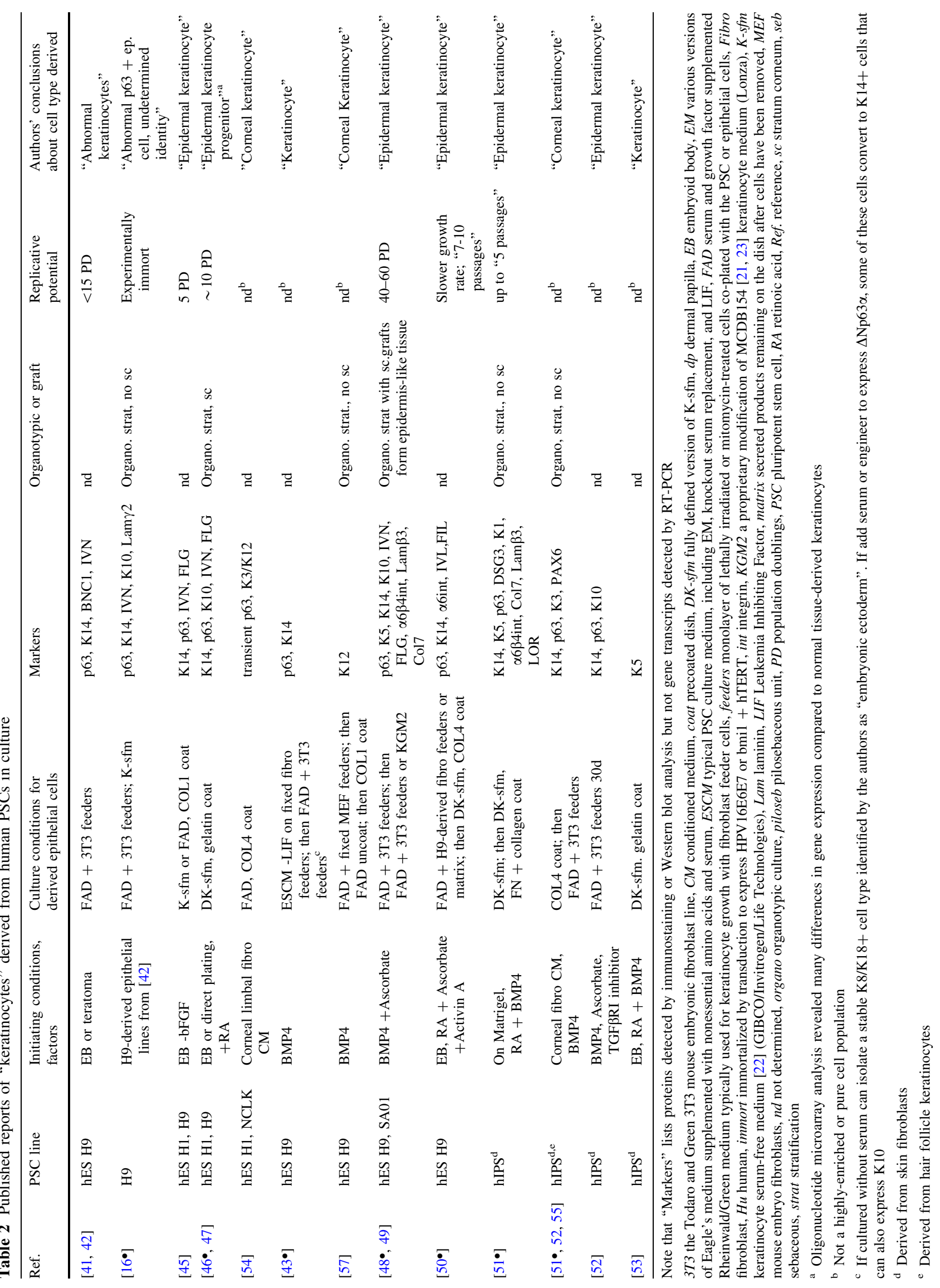


$[35,36]$. The basis for their approach was the prior observation [37] that a variety of developmental lineages form in culture inside aggregates of mES cells termed "embryoid bodies (EBs)". ES cells are encouraged to aggregate into EBs by suspending them in a small hanging drop of culture medium. In the Bagutti studies, EBs allowed to attach and spread on gelatin-coated dishes yielded some K14+ and a6ß4int+ cells within several weeks. Kawasaki, Sasai, and colleagues [38] advanced this field as part of a study aimed at generating neural cells from mES. They found that BMP4 suppressed neurectoderm commitment of mES cells, thereby diverting more cells toward epithelial lineages including some K14+ cells. This study [38] also discovered that prior EB formation was not essential but that $\mathrm{K} 14+$ cell frequency was increased by plating $\mathrm{mES}$ cells on living or fixed fibroblast or bone marrow stromal cell feeder cells.

Coraux, Aberdam, and colleagues [39, 40] reported formation of both epidermal- and connective tissue-like cells by plating mES cells pretreated with BMP4 or ascorbate onto a dish coated with proteins secreted by fibroblasts. When plated at the air-liquid interface, the heterogenous mES-derived cell population sorted into a stratified epithelial layer expressing many markers of epidermal differentiation and an underlying connective tissuelike layer.

\section{Human ES-Derived Epithelial Cells}

Green, Iuchi, and colleagues published the first report that human ES (hES) cells can produce epithelial cells that express p63 and K14 and that can stratify and express IVN [41]. These cells were noted to have an unusually limited proliferative potential compared to somatic tissue-derived keratinocytes but they could be immortalized if engineered to evade both $\mathrm{p} 16^{\mathrm{INK} 4 \mathrm{~A}}$ - and telomere-related senescence barriers [16•, 42]. Their colony morphology in the FAD + 3T3 feeder system differed from that of somatic keratinocytes. The authors concluded that these cells were abnormal keratinocyte-like cells [42]. This was investigated more thoroughly by Dabelsteen, Rheinwald, and colleagues [16•], who determined that the cells had abnormal growth regulatory mechanisms and concluded that their identity could be any of the many p63+ cell types.

Medawar, Aberdam, and colleagues [43•, 44] identified a stable, potential developmental intermediate during conversion of ES cells to p63/K14+ cells. mES or hES cells plated on a fixed fibroblast layer and treated for several days with BMP4 in the absence of serum produced many $\mathrm{K} 8 / \mathrm{K} 18+$ epithelial cells that lacked pluripotency markers and could be serially passaged. The authors proposed that these were embryonic ectodermal cells. If treated with serum or engineered to express $\Delta \mathrm{Np} 63 \alpha$, some of these cells began expressing K14 and could stratify to form $\mathrm{K} 10+$ cells.

Metallo, Ji, Palacek, and colleagues [45-47] identified retinoic acid (RA) as an alternative to BMP4 as an agent to increase the frequency of hES-derived epithelial cells expressing p63, K14, IVN, K10, and Lam $\gamma 2$. In organotypic culture these cells formed a stratified, epidermal-like epithelium. Importantly, these cells were found to have a gene transcription pattern clearly different from, although sharing many key features with, normal somatic epidermal keratinocytes.

Guenou, Nissan, Baldeschi, and colleagues [48•, 49] plated clumps of hES cells into the FAD + 3T3 feeder system supplemented with BMP4+ ascorbate. After 40 days, $40-50 \%$ of the cells had formed K14+ clusters with morphology reminiscent of keratinocytes. These were isolated and expanded by subculture either in the FAD + 3T3 feeder system or in semi-defined medium. They were found to express many markers of epidermal keratinocytes and to form an epidermal-like epithelium in organotypic culture and in grafts to immunodeficient mice. The authors stated, although without showing serial growth data, that these cells possessed a replicative lifespan of 40-60 PD, similar to that of somatic tissue-derived keratinocytes. This is notable since PSC-derived, keratinocytelike cells produced by other labs consistently displayed a very short lifespan of 5-15 PD (Table 2).

Kidwai, Cao, and colleagues [50•] found that addition of Activin A for a specific 3 day window of time markedly increased the yield of p63+ cells from hES cells at the expense of cells that express the neurectoderm marker PAX6.

\section{Derivation of Epidermal Keratinocyte-like Cells} from iPS Cells

Recently several groups have derived epithelial cells displaying properties of keratinocytes from iPS cells generated from skin fibroblasts. Bilousova, Roop, and colleagues [28.] formed EBs from murine iPS (miPS) cells and treated them with RA. These were then plated on COL4-coated dishes, treated with BMP4 for 3 days, and the medium then changed to DK-sfm for 6 days. At this time many cells that had migrated out of the attached EBs were p63/K14+. EBs were selectively removed and the remaining cell population was progressively enriched for $\mathrm{K} 14+$ cells by serial culture in DK-sfm on COL4-coated dishes. The cells could express $\mathrm{K} 1$ and Loricrin (LOR) and had a replicative lifespan of $\sim 18 \mathrm{PD}$, similar to that of newborn murine somatic epidermal keratinocytes cultured in the same conditions. This study was unique in demonstrating that miPS-derived p63/K14+ cells could form a stratified epidermis as well as hair follicles and sebaceous glands when 
grafted onto the skin as a cell suspension mixed with normal skin fibroblast and dermal papilla cells.

Itoh, Christiano, and colleagues [51•] plated clumps of hiPS cells on Matrigel-coated dishes in DK-sfm supplemented with RA and BMP4 for the first 4 days. After 30 days, $45 \%$ of the cells were $\mathrm{K} 14+$, increasing to $70 \%$ after subculture to coated dishes in DK-sfm, and some cells expressed DSG3 and K1. In organotypic culture, the cells stratified and expressed suprabasal K1 and LOR. Subsequent studies by others [51 $, 52,53]$ have reported derivation of p63/K5/K14+ cells from hiPS cells bearing a variety of inherited mutations that have a severe epidermal phenotype.

Research Aimed at Generating Corneal Keratinocytes from PSC Cells

Several groups have sought to derive corneal keratinocytes from hES cells by attempting to direct lineage commitment by early exposure to products secreted by corneal fibroblasts. Ahmad, Lako, and colleagues [54] plated clumps of hES cells on COL4-coated dishes in medium conditioned by human corneal limbal fibroblasts. They observed a transient generation of $\mathrm{p} 63+$ and $\mathrm{K} 3 / \mathrm{K} 12+$ cells peaking at 1 week but such cells were not sustained in the population. Shalom-Feuerstein, Aberdam, Petit, and colleagues [55] generated hiPS cells from hair follicle keratinocytes and skin fibroblasts and plated these in corneal fibroblast conditioned medium (CM) with a 4 day BMP4 treatment. 2 weeks later, many cells appeared by immunostaining and FACS analysis to coexpress p63, K14, K3, and PAX6. PAX6 was proposed by the authors as a marker of corneal epithelial identity but, in fact, is expressed by early neuroectoderm and many mature neurons as well as being essential for development of all structures of the eye [56]. These cells formed a bilayer in organotypic culture with suprabasal K3 expression. Hewitt, Aberdam, Garlick, and colleagues [57] used a complex multistep approach involving a possible ectoderm-like intermediate cell line, but without attempting to direct cells into a corneal lineage. They generated epithelial cells that stratified and produced some suprabasal $\mathrm{K} 12+$ cells. These studies are intriguing but none compared the effects of dermal versus corneal fibroblast $\mathrm{CM}$ or looked for expression of the epidermal keratin pair $\mathrm{K} 1 / \mathrm{K} 10$, nor were homogenous and serially cultivable cell populations obtained.

\section{Critique}

The literature to date permits a number of important conclusions. The common feature of successful protocols is plating PSCs as aggregates into culture conditions permissive for growth of keratinocytes and other members of the p63 family of epithelial cell types (e.g., the FAD + fibroblast feeder system or a semi-defined or defined keratinocyte medium). Then, provided that there is sufficient space on the dish to permit outward migration and further proliferation, during the following 1-4 weeks the PSCs lose pluripotency markers and some cells begin expressing p63 and K14. Prior EB formation by PSC does not enhance generation of p63/K14+ cells. No inducers of PSC commitment are essential as medium additives to generate such cells. However, RA, BMP4, Activin A, ascorbate, and TGF $\beta$ receptor inhibitor, either individually or in various combinations, increase the frequency of p63/ $\mathrm{K} 14+$ cells. An intermediate state, in which pluripotency markers no longer are expressed but K8/K18 expression is increased, may precede generation of p63/K14+ cells. As yet there is no evidence that conversion can be directed toward a specific keratinocyte subtype (e.g., cornea) by use of region-specific fibroblasts or their products. Derived p63/K14+ cells typically also express $\alpha 6 \beta 4$ integrin, Laminin-332, and K5, the partner of K14 for filament formation, although none of these markers are specific to keratinocytes. The replicative lifespan of PSC-derived p63/ $\mathrm{K} 14+$ cells typically is much shorter and the growth rate slower than that of tissue-derived somatic keratinocytes. However, two studies have reported replicative lifespans of miPS-derived and hES-derived p63/K14+ cells equivalent to that of normal keratinocytes [28•, 48•].

The stated objective of all the above studies was to derive keratinocytes from PSCs. Many studies using human PSCs claim to have achieved the goal of generating keratinocytes useful for clinical tissue replacement. Almost all of these studies mistakenly used expression of p63, $\mathrm{K} 14, \alpha 6 \beta 4$ integrin, Laminin-332, and K5 as evidence that the proliferative cells they obtained were basal layer keratinocytes. The K5/K14 keratin pair (originally termed the $58 \mathrm{kd}$ and $50 \mathrm{kd}$ keratins for their electrophoretic mobility) have been known since the early 1980s to be expressed by the basal, proliferative cells of many epithelial tissues, including mammary gland, tracheobronchus, and urothelium in addition to the epidermis and other stratified squamous epithelia $[15,29,58] . \alpha 6 \beta 4$ integrin and its cognate basal lamina protein ligand Laminin-332 also are expressed by the basal cells of these epithelia. The discovery of p63 in the late 1990s included the observation that this transcriptional regulator is expressed by the basal cell compartments of all of the above-mentioned epithelia and that knockout of p63 in mice results in failure to develop all of these epithelia [59-61].

Members of this large family of p63/K14+ cell types arise from all three embryonic germ layers (Fig. 1). The various keratinocyte subtypes that form different stratified squamous epithelia arise from all three embryonic germ layers. Thus, PSCs are not obliged to convert first to an 
ectodermal precursor in order to undergo further commitment to a keratinocyte lineage. Interestingly, soon after the development of the FAD + 3T3 feeder system to culture epidermal keratinocytes it was found that this system also is ideal for clonal growth and serial propagation of all epithelial cell types now known to express p63 [15, 17, 20]. K-sfm and other semi-defined "keratinocyte" media also prove to promote excellent growth of all p63 epithelial cell types in addition to p63+ hES-derived epithelial cells (e.g., $[16 \bullet, 25]$. Thus, expression of p63 and markers of keratinocyte basal cells does not support the conclusion that PSC cells have converted to a keratinocyte lineage.

Many studies identified in their PSC-derived, p63/K14+ populations stratifying cells expressing IVL, K1/K10, and other epidermal terminal differentiation proteins. In normal epithelia in vivo, this would narrow down identification to epidermis, exocervix, and thymus, with occasional expression in gingiva and hard palate [62]. An organotypic culture system is widely used to assess the ability of cells to undergo epidermoid differentiation. This system was initially optimized to promote complete epidermoid differentiation by serially cultured primary human epidermal keratinocytes [34]. However, in this system other keratinocyte subtypes, such as the non-keratinizing buccal and floor of mouth mucosal keratinocytes, abnormally express suprabasal K1/ K10 [33], despite the fact that keratinocytes retain their subtype identity during serial culture and ability to regenerate their appropriate pattern of suprabasal differentiation without specific stromal instruction in xenograft transplants [18, 19]. The study by Dabelsteen, Rheinwald, and colleagues [16•] found that somatic tissue-derived human urothelial and tracheobronchial epithelial cells stratify and form an epidermal-like stratum corneum with suprabasal K1/K10 and IVL in organotypic culture. Even human prostate epithelial cells undergo epidermoid differentiation in organotypic culture (W. Wei, P. Barron, and J. Rheinwald, unpublished). Mindful that chronic Vitamin A (RA) deprivation in vivo causes reversible epidermoid differentiation of many p63+ family epithelial tissues [63, 64], we tried adding RA to the medium but found that p63+ epithelial cell types continued to display features of epidermoid differentiation in organotypic culture [16•,33]. This result precludes the use of standard organotypic assays, or of the occasional expression of IVL or K1/K10 by stratifying cells in conventional culture, as evidence of epidermal keratinocyte identity. This issue is especially relevant for evaluating studies in which an appropriate variety of other normal epithelial cell types is not compared to the PSC-derived cell for histogenesis and marker expression. The strongest evidence for epidermal identity has been obtained in several studies that demonstrated formation of an epidermal-like tissue by PSC-derived epithelial cells xenografted to the skin (Tables 1 and 2). However, the extent to which other p63+ epithelial cell types may change their differentiation to more epidermoid character in response to ectopic transplantation to the skin surface remains to be determined.

Most studies reporting PSC to keratinocyte-like cell conversion have focused on demonstrating expression of cell type and differentiation markers. However, this is only one of two essential criteria for generation of an authentic and useful somatic cell type. The other is whether the PSCderived cell displays growth regulatory mechanisms characteristic of the normal somatic cell in question, which can help to both confirm identity or close similarity to the tissue-derived cell type and predict whether they would behave normally as tissue stem cells in transplants. Our study [16•] examining the growth properties of three clonally isolated, hES-derived p63/K14+ cell lines revealed that they grew less well in K-sfm and were less dependent on EGF and less sensitive to growth inhibition by serum than normal keratinocytes or urothelial cells. PSC-derived cells, even if able to undergo the desired differentiation, may not be suitable for transplantation if they lack normal growth regulatory systems.

With the exception of one report [48•], the p63/K14+ epithelial cells generated from human PSCs in all studies displayed a very short replicative lifespan, precluding isolation as pure populations as well as potential use for epidermal tissue regeneration. Differences in the starting PSC cells or in the protocols may yield cells that differ with

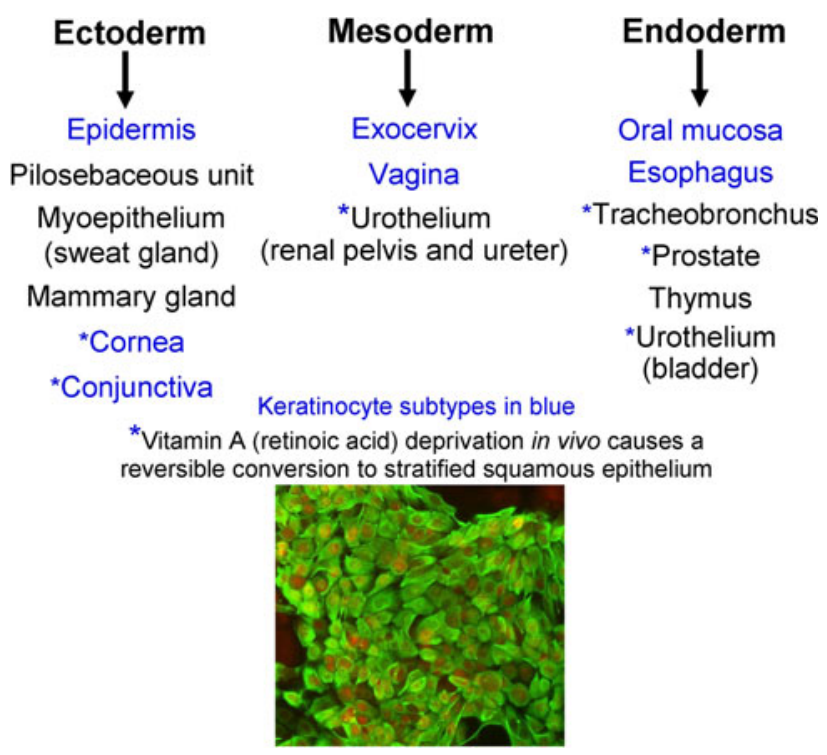

Fig. 1 Embryonic germ layer origin of p63-expressing epithelial cell types and sensitivity of their differentiation to retinoid deprivation in vivo. Keratinocyte subtypes are shown in blue font. Asterisks indicate those epithelia for which Vitamin A [retinoic acid (RA)] deprivation in vivo causes a reversible conversion to an epidermoid, stratified squamous epithelial form of differentiation. Panel shows an $\mathrm{H} 9$ hES-derived epithelial colony (hESderK clone 2A2/E6E7 [16•, 42]) immunostained for p63 (red) and K14 (green) (Color figure online) 
respect to cell type or degree of "normality". Protocols that do not include clonal isolation or other very rigorous purification of p63/K14+ cells may maintain some K8/K18+ ectoderm- or endoderm-like precursor cells in the population during serial passage. Such cells were noted to have a long lifespan and the potential to convert spontaneously to p63/K14+ cells $[43 \cdot, 44]$, the result of which would be an apparently long lifespan of the p63/K14+ cells.

\section{Future Directions}

Many groups have established that it is possible, using a variety of protocols and facilitating factors, to generate p63/K14+ epithelial cells having the potential to undergo epidermoid differentiation. Effort now should be devoted to understanding the PSC-to-stratified epithelial cell process in more detail. The time course and quantitation of conversion under various conditions should be compared by disaggregating cultures to single cells and plating in the FAD + 3T3 feeder system to identify cells able to form epithelial colonies of at least a size of 1,000 cells $(\sim 10$ PD). Such colonies can be immunostained for p63, K14, and other markers, or to look for loss of Oct 4 , expression of $\mathrm{K} 8$, etc. Some colonies can be isolated at different time points after beginning the conversion protocol to expand for further analysis, experimentally immortalizing them if necessary to permit this (e.g., [16•, 42]).

The stable, propagable $\mathrm{K} 8 / \mathrm{K} 18+$ epithelial cells that can be derived from mES and hES cells $[43 \cdot, 44]$ should be examined more thoroughly to determine their identity and similarity to early developmental intermediates in vivo. The conclusion that these cells are embryonic ectoderm is premature. K8/K18 were first discovered as "Endo A and Endo B"-cytokeratins of early parietal and visceral endoderm [65] and BMP4 not only suppresses neurectoderm formation from ectoderm but also promotes formation of endoderm from ES cells [66]. Furthermore, although RA is used in some protocols aimed at generating epidermal keratinocytes, this agent was first noted to convert mouse embryonal carcinoma and ES cells toward an early endoderm phenotype [67]. It would be interesting to learn whether PSC-derived, embryonic endoderm-like cells preferentially produce p63/K14+ cell types that normally arise from endoderm during development (see Fig. 1). Related to this topic, whether and how PSC conversion can be directed toward a specific p63+ epithelial cell type remains a major question. Although postnatal somatic keratinocytes do not require signaling from their regional underlying connective tissue to differentiate appropriately in xenograft systems $[18,19]$, acquisition of specific cell type identity (e.g., corneal, tracheobronchial, urothelial) during development may require exposure to regional connective tissue fibroblasts. Studies should compare dermal versus other connective tissue fibroblasts and embryonic versus adult for such inductive activity.

Accurate identification of PSC-derived, p63/K14+ cells and determination of how closely they resemble normal somatic epithelial cells will be aided by microarray analysis of gene expression on pure, homogenous cell populations. An initial study [46•] of an uncloned hES-derived population greatly enriched for $\mathrm{K} 14+$ cells revealed many differences between these cells and normal primary epidermal keratinocytes. PSC-derived populations may be mixtures of several p63+ cell types or otherwise heterogenous with respect to gene expression. Clonally isolated populations should be compared to epidermal keratinocytes and a variety of normal tissue-derived p63+ cell types. Growth requirements in culture, including mitogendependence and inhibitor sensitivity, also are excellent bellwethers of normality [16॰]. When cultured in semidefined media, all normal p63+ epithelial cell types require either EGF, KGF, or HGF for growth [25] and are strongly growth inhibited by TGF $\beta$ [68, 69] and by serum [25]. Organotypic and transplantation assays used to determine cell type identity should be permissive for various types of epithelial differentiation and not push all cells toward epidermoid differentiation. The internal skin flap xenograft system $[18,19,70]$ is one example of a permissive system.

\section{Conclusions}

There is a wonderful quote by the author Louis Lamour: "There will come a time when you believe that everything is finished. That will be the beginning." As described in this review, despite much progress this field is still very much in an initial, discovery phase. Awareness that many p63+cell types may be arising from PSC cells expands the opportunities for discovery immensely. PSC-derived, p63/ K14+ cells now should be characterized more precisely and their normality examined more critically using better growth and differentiation assays and global gene expression profiling. Protocols should be compared quantitatively and qualitatively for frequency and identity of cells generated. Progress would be aided immensely by free exchange of PSCs and derived p63/K14+ cell lines among laboratories. Continued research on this subject will expand our understanding of the essential factors for embryonic development of the many keratinocyte subtypes and other p63+ epithelial cell types.

Disclosure J.G. Rheinwald declares that he has no conflict of interest. 


\section{References}

Papers of particular interest, published recently, have been highlighted as:

- Of importance

1. Evans MJ, Kaufman MH (1981) Establishment in culture of pluripotential cells from mouse embryos. Nature 292:154-156

2. Martin GR (1981) Isolation of a pluripotent cell line from early mouse embryos cultured in medium conditioned by teratocarcinoma stem cells. Proc Natl Acad Sci USA 78:7634-7638

3. Shamblott MJ, Axelman J, Wang S et al (1998) Derivation of pluripotent stem cells from cultured human primordial germ cells. Proc Natl Acad Sci USA 95:13726-13731

4. Thomson JA, Itskovitz-Eldor J, Shapiro SS et al (1998) Embryonic stem cell lines derived from human blastocysts. Science 282:1145-1147

5. Lin SL (2011) Concise review: deciphering the mechanism behind induced pluripotent stem cell generation. Stem Cells 29:1645-1649

6. Takahashi K, Tanabe K, Ohnuki M et al (2007) Induction of pluripotent stem cells from adult human fibroblasts by defined factors. Cell 131:861-872

7. Takahashi K, Yamanaka S (2006) Induction of pluripotent stem cells from mouse embryonic and adult fibroblast cultures by defined factors. Cell 126:663-676

8. Yu J, Vodyanik MA, Smuga-Otto K et al (2007) Induced pluripotent stem cell lines derived from human somatic cells. Science 318:1917-1920

9. Robinton DA, Daley GQ (2012) The promise of induced pluripotent stem cells in research and therapy. Nature 481:295-305

10. Compton CC, Gill JM, Bradford DA et al (1989) Skin regenerated from cultured epithelial autografts on full-thickness burn wounds from 6 days to 5 years after grafting. A light, electron microscopic and immunohistochemical study. Lab Investig 60:600-612

11. Gallico GG 3rd, O'Connor NE, Compton CC et al (1984) Permanent coverage of large burn wounds with autologous cultured human epithelium. N Engl J Med 311:448-451

12. Pellegrini G, Traverso CE, Franzi AT et al (1997) Long-term restoration of damaged corneal surfaces with autologous cultivated corneal epithelium. Lancet 349:990-993

13. Rheinwald JG, Green H (1975) Serial cultivation of strains of human epidermal keratinocytes: the formation of keratinizing colonies from single cells. Cell 6:331-343

14. Watt FM, Green H (1981) Involucrin synthesis is correlated with cell size in human epidermal cultures. J Cell Biol 90:738-742

15. Wu YJ, Parker LM, Binder NE et al (1982) The mesothelial keratins: a new family of cytoskeletal proteins identified in cultured mesothelial cells and nonkeratinizing epithelia. Cell 31: 693-703

16. • Dabelsteen S, Hercule P, Barron P, et al. (2009) Epithelial cells derived from human embryonic stem cells display $\mathrm{p} 16^{\mathrm{INK} 4 \mathrm{~A}}$ senescence, hypermotility, and differentiation properties shared by many p63+ somatic cell types. Stem Cells 27:1388-1399. This paper identified growth control abnormalities in hES-derived, p63/ K14+ cells and showed that the pattern of differentiation these cells display in organotypic culture is similar to that of many p63 + epithelial cell types and not only epidermal keratinocytes

17. Lechner JF, Haugen A, Autrup H et al (1981) Clonal growth of epithelial cells from normal adult human bronchus. Cancer Res 41:2294-2304

18. Lindberg K, Brown ME, Chaves HV et al (1993) In vitro propagation of human ocular surface epithelial cells for transplantation. Investig Ophthalmol Vis Sci 34:2672-2679
19. Lindberg K, Rheinwald JG (1990) Three distinct keratinocyte subtypes identified in human oral epithelium by their patterns of keratin expression in culture and in xenografts. Differentiation 45:230-241

20. Taylor-Papadimitriou J, Shearer M, Stoker MG (1977) Growth requirements of human mammary epithelial cells in culture. Int $\mathrm{J}$ Cancer 20:903-908

21. Boyce ST, Ham RG (1983) Calcium-regulated differentiation of normal human epidermal keratinocytes in chemically defined clonal culture and serum-free serial culture. J Investig Dermatol $81: 33 \mathrm{~s}-40 \mathrm{~s}$

22. Pirisi L, Yasumoto S, Feller M et al (1987) Transformation of human fibroblasts and keratinocytes with human papillomavirus type 16 DNA. J Virol 61:1061-1066

23. Shipley GD, Pittelkow MR (1987) Control of growth and differentiation in vitro of human keratinocytes cultured in serumfree medium. Arch Dermatol 123:1541a-1544a

24. Rice RH, Green H (1979) Presence in human epidermal cells of a soluble protein precursor of the cross-linked envelope: activation of the cross-linking by calcium ions. Cell 18:681-694

25. Wei W, Barron PD, Rheinwald JG (2010) Modulation of TGFbeta-inducible hypermotility by EGF and other factors in human prostate epithelial cells and keratinocytes. In vitro Cell Dev Biol Animal 46:841-855

26. Hennings H, Michael D, Cheng C et al (1980) Calcium regulation of growth and differentiation of mouse epidermal cells in culture. Cell 19:245-254

27. Nowak JA, Fuchs E (2009) Isolation and culture of epithelial stem cells. Methods Mol Biol 482:215-232

28. • Bilousova G, Chen J, Roop DR (2011) Differentiation of mouse induced pluripotent stem cells into a multipotent keratinocyte lineage. J Investig Dermatol 131:857-864. This paper describes a protocol for routine generation from miPS cells of p63/ $K 14+$ cells possessing a replicative lifespan similar to that of normal primary murine keratinocytes and the ability to form epidermis, hair follicles, and sebaceous glands in vivo when transplanted admixed with skin fibroblasts and dermal papilla cells

29. Sun TT, Eichner R, Nelson WG et al (1983) Keratin classes: molecular markers for different types of epithelial differentiation. J Investig Dermatol 81:109s-115s

30. O'Guinn WM, Galvin S, Schermer A et al (1987) Patterns of keratin expression define distinct pathways of epithelial development and differentiation. Curr Top Dev Biol 22:97-125

31. Mehrel T, Hohl D, Rothnagel JA et al (1990) Identification of a major keratinocyte cell envelope protein, loricrin. Cell 61: 1103-1112

32. Lynley AM, Dale BA (1983) The characterization of human epidermal filaggrin. A histidine-rich, keratin filament-aggregating protein. Biochim Biophys Acta 744:28-35

33. Schön M, Rheinwald JG (1996) A limited role for retinoic acid and retinoic acid receptors RAR $\alpha$ and $\operatorname{RAR} \beta$ in regulating keratin 19 expression and keratinization in oral and epidermal keratinocytes. J Investig Dermatol 107:428-438

34. Parenteau NL, Nolte CM, Bilbo P et al (1991) Epidermis generated in vitro: practical considerations and applications. J Cell Biochem 45:245-251

35. Bagutti C, Hutter C, Chiquet-Ehrismann R et al (2001) Dermal fibroblast-derived growth factors restore the ability of $\beta 1$ integrin-deficient embryonal stem cells to differentiate into keratinocytes. Dev Biol 231:321-333

36. Bagutti C, Wobus AM, Fassler R et al (1996) Differentiation of embryonal stem cells into keratinocytes: comparison of wild-type and $\beta 1$ integrin-deficient cells. Dev Biol 179:184-196

37. Doetschman TC, Eistetter H, Katz M et al (1985) The in vitro development of blastocyst-derived embryonic stem cell lines: 
formation of visceral yolk sac, blood islands and myocardium. J Embryol Exp Morphol 87:27-45

38. Kawasaki H, Mizuseki K, Nishikawa S et al (2000) Induction of midbrain dopaminergic neurons from ES cells by stromal cellderived inducing activity. Neuron 28:31-40

39. Aberdam D (2004) Derivation of keratinocyte progenitor cells and skin formation from embryonic stem cells. Int J Dev Biol 48:203-206

40. Coraux C, Hilmi C, Rouleau M et al (2003) Reconstituted skin from murine embryonic stem cells. Curr Biol 13:849-853

41. Green H, Easley K, Iuchi S (2003) Marker succession during the development of keratinocytes from cultured human embryonic stem cells. Proc Natl Acad Sci USA 100:15625-15630

42. Iuchi S, Dabelsteen S, Easley K et al (2006) Immortalized keratinocyte lines derived from human embryonic stem cells. Proc Natl Acad Sci USA 103:1792-1797

43. - Aberdam E, Barak E, Rouleau M, et al. (2008) A pure population of ectodermal cells derived from human embryonic stem cells. Stem Cells 26:440-444. This paper identified a stable K8/ $K 18+$ epithelial cell type derived from hES cells that has the potential to spontaneously, or if engineered to express $\triangle N p 63 \alpha$, express $\mathrm{K} 14$

44. Medawar A, Virolle T, Rostagno P et al (2008) $\Delta$ Np63 is essential for epidermal commitment of embryonic stem cells. PLoS ONE 3:e3441

45. Ji L, Allen-Hoffmann BL, de Pablo JJ et al (2006) Generation and differentiation of human embryonic stem cell-derived keratinocyte precursors. Tissue Eng 12:665-679

46. - Metallo CM, Azarin SM, Moses LE, et al. (2010) Human embryonic stem cell-derived keratinocytes exhibit an epidermal transcription program and undergo epithelial morphogenesis in engineered tissue constructs. Tissue Eng Part A 16:213-223. This paper was the first to identify substantial differences in global gene transcription between hES-derived p63/K14 + cells and normal primary human epidermal keratinocytes

47. Metallo CM, Ji L, de Pablo JJ et al (2008) Retinoic acid and bone morphogenetic protein signaling synergize to efficiently direct epithelial differentiation of human embryonic stem cells. Stem Cells 26:372-380

48. - Guenou H, Nissan X, Larcher F, et al. (2009) Human embryonic stem-cell derivatives for full reconstruction of the pluristratified epidermis: a preclinical study. Lancet 374:1745-1753. This paper is the only report to date of generating long replicative lifespan p63/K14 + cells from hES cells in culture

49. Nissan X, Denis JA, Saidani M et al (2011) miR-203 modulates epithelial differentiation of human embryonic stem cells towards epidermal stratification. Dev Biol 356:506-515

50. - Kidwai FK, Liu H, Toh WS, et al. (2013) Differentiation of human embryonic stem cells into clinically amenable keratinocytes in an autogenic environment. J Invest Dermatol 133:618-628. This paper describes a thorough comparison of several protocols for producing p63/K14+ cells from hES cells and identifies strong positive effects of Activin A

51. - Itoh M, Kiuru M, Cairo MS, et al. (2011) Generation of keratinocytes from normal and recessive dystrophic epidermolysis bullosa-induced pluripotent stem cells. Proc Natl Acad Sci USA 108:8797-8802. This paper is the first report of routine generation of p63/K14 + cells from human iPS cells

52. Shalom-Feuerstein R, Serror L, Aberdam E et al (2013) Impaired epithelial differentiation of induced pluripotent stem cells from ectodermal dysplasia-related patients is rescued by the small compound APR-246/PRIMA-1MET. Proc Natl Acad Sci USA 110:2152-2156
53. Tolar J, Xia L, Lees CJ et al (2013) Keratinocytes from induced pluripotent stem cells in junctional epidermolysis bullosa. J Investig Dermatol 133:562-565

54. Ahmad S, Stewart R, Yung S et al (2007) Differentiation of human embryonic stem cells into corneal epithelial-like cells by in vitro replication of the corneal epithelial stem cell niche. Stem Cells 25:1145-1155

55. Shalom-Feuerstein R, Serror L, De La Forest Divonne S et al (2012) Pluripotent stem cell model reveals essential roles for miR-450b-5p and miR-184 in embryonic corneal lineage specification. Stem Cells 30:898-909

56. Simpson TI, Price DJ (2002) Pax6; a pleiotropic player in development. BioEssays 24:1041-1051

57. Hewitt KJ, Shamis Y, Carlson MW et al (2009) Three-dimensional epithelial tissues generated from human embryonic stem cells. Tissue Eng Part A 15:3417-3426

58. Moll R, Franke WW, Schiller DL et al (1982) The catalog of human cytokeratins: patterns of expression in normal epithelia, tumors and cultured cells. Cell 31:11-24

59. Mills AA, Zheng B, Wang XJ et al (1999) p63 is a p53 homologue required for limb and epidermal morphogenesis. Nature 398:708-713

60. Yang A, Kaghad M, Wang Y et al (1998) p63, a p53 homolog at 3q27-29, encodes multiple products with transactivating, deathinducing, and dominant-negative activities. Mol Cell 2:305-316

61. Yang A, Schweitzer R, Sun D et al (1999) p63 is essential for regenerative proliferation in limb, craniofacial and epithelial development. Nature 398:714-718

62. Moll R, Divo M, Langbein L (2008) The human keratins: biology and pathology. Histochem Cell Biol 129:705-733

63. Wolbach SB, Howe PR (1925) Tissue changes following deprivation of fat-soluble A vitamin. J Exp Med 42:753-777

64. Wolbach SB, Howe PR (1933) Epithelial repair in recovery from vitamin A deficiency : an experimental study. J Exp Med 57:511-526

65. Howe WE, Klier FG, Oshima RG (1986) Murine endodermal cytokeratins Endo A and Endo B are localized in the same intermediate filament. J Histochem Cytochem 34:785-793

66. Conley BJ, Ellis S, Gulluyan L et al (2007) BMPs regulate differentiation of a putative visceral endoderm layer within human embryonic stem-cell-derived embryoid bodies. Biochimie Biologie Cellulaire 85:121-132

67. Soprano DR, Teets BW, Soprano KJ (2007) Role of retinoic acid in the differentiation of embryonal carcinoma and embryonic stem cells. Vitam Horm 75:69-95

68. Rollins BJ, O'Connell TM, Bennett G et al (1989) Environmentdependent growth inhibition of human epidermal keratinocytes by recombinant human TGF $\beta$. J Cell Physiol 139:455-462

69. Shipley GD, Pittelkow MR, Wille JJ Jr et al (1986) Reversible inhibition of normal human prokeratinocyte proliferation by type $\beta$ transforming growth factor-growth inhibitor in serum-free medium. Cancer Res 46:2068-2071

70. Barrandon Y, Li V, Green H (1988) New techniques for the grafting of cultured human epidermal cells onto athymic animals. J Investig Dermatol 91:315-318

71. Troy TC, Turksen K (2005) Commitment of embryonic stem cells to an epidermal cell fate and differentiation in vitro. Dev Dyn 232:293-300

72. Haase I, Knaup R, Wartenberg M et al (2007) In vitro differentiation of murine embryonic stem cells into keratinocyte-like cells. Eur J Cell Biol 86:801-805

73. Huang HJ, Gao QS, Tao BF et al (2008) Long-term culture of keratinocyte-like cells derived from mouse embryonic stem cells. In Vitro Cell Dev Biol 44:193-203 